\title{
A eficácia do molnupiravir no tratamento em paciente com Covid-19: revisão
}

\section{sistemática}

\author{
The effectiveness of molnupiravir for treatment in patients with Covid-19: a systematic review
}

La eficacia del molnupiravir para el tratamiento de pacientes con Covid-19: una revisión

\section{sistemática}

\author{
Wanderson Silva Macedo de Sousa \\ ORCID: https://orcid.org/0000-0002-4474-5667 \\ Centro Universitário Uninovafapi, Brasil \\ E-mail: wandersonsousa8@hotmail.com \\ Ana Claudia de Miranda Adad \\ ORCID: https://orcid.org/0000-0002-6257-3790 \\ Centro Universitário Uninovafapi, Brasil \\ E-mail: anacadad10@gmail.com \\ Rafaelly dos Santos \\ ORCID: https://orcid.org/0000-0002-3622-9662 \\ Universidade Federal de Sergipe, Brasil \\ E-mail: rafaellysantos.01@gmail.com \\ Jonatas Paulino da Cunha Monteiro Ribeiro \\ ORCID: https://orcid.org/0000-0002-0106-1345 \\ Universidade Federal do Piauí, Brasil \\ E-mail: jonatasmonteirofnt@gmail.com \\ Tayane de Jesus Bispo \\ ORCID: https://orcid.org/0000-0003-2246-188X \\ Universidade Federal de Sergipe, Brasil \\ E-mail: tayane99@academico.ufs.br \\ Manoela de Moura Gervazoni \\ ORCID: https://orcid.org/0000-0002-6571-9265 \\ Centro Universitário da Fundação Assis Gurgacz, Brasil \\ E-mail: mmgervazoni@minha.fag.edu.br \\ Gabriela Vieira Pontalti \\ ORCID: https://orcid.org/0000-0002-0182-0612 \\ Universidade de Caxias do Sul, Brasil \\ E-mail: gvpontalti@ucs.br \\ Giovanna Vargas Haendchen \\ ORCID: https://orcid.org/0000-0002-7783-6573 \\ Universidade de Caxias do Sul, Brasil \\ E-mail: gvhaendchen@ucs.br \\ Gabriela Chmilouski \\ ORCID: https://orcid.org/0000-0002-2366-0867 \\ Centro Universitário da Fundação Assis Gurgacz Brasil \\ E-mail: gabrielachmilouski@ outlook.com \\ Walberth Gabriel Cardoso dos Santos \\ ORCID: https://orcid.org/0000-0002-5815-3830 \\ Universidade Federal do Piauí, Brasil \\ E-mail: walberthcardoso@gmail.com
}

\begin{abstract}
Resumo
No início de 2020, o surto de (SARS-CoV-2), causador da Covid-19, disseminou-se rapidamente pelo mundo, tanto que a Organização Mundial da Saúde (OMS) declarou estado de pandemia. Houve uma grande preocupação, pois ainda não se tinha conhecimento sobre essa nova patologia e os países não tinham um plano de saúde estratégico. Diante disso, este artigo tem o objetivo de verificar a eficácia do molnupiravvir em estudos clínicos. Trata-se de uma de revisão sistemática, utilizou-se do protocolo Preferred Reporting Items for Systematic Reviwes and a MetaAnalyses (PRISMA). A estratégia de busca foi construída através dos descritores controlados do Medical Subject Headings (MeSH) e dos recursos dos Descritores em Ciência da Saúde (DeCS). As combinações dos descritores resultaram nos seguintes termos: "Molnupiravir", "Covid-19" e "Sars-cov-2". Ademais, foram aplicados os operadores booleanos "OR" e "AND”. As bases de dados utilizadas foram Medline, Lilacs, ambas via Biblioteca Virtual da Saúde (BVS); PUBMED; Scientific Eletronic Library Online (Scielo); Science Direct e Google Scholar. O molnupiravir pode ser usada como medida de profilaxia, sendo assim, evitando a hospitalização, já em pacientes hospitalizados e que possuíam comorbidades, o medicamento não possui efeito benéfico clínico. Como as amostras
\end{abstract}


nos estudos, em média, foram baixas, é necessário que mais estudos continuem sendo feitos e reproduzidos para comprovar se o molnupiravir possui eficácia no combate à Covid-19.

Palavras-chave: Covid-19; Molnupirair; SARS-CoV-2.

\begin{abstract}
In early 2020, the outbreak of (SARS-CoV-2), the vírus that causes Covid-19, spread rapidly around the world, so much so that the World Health Organization (WHO) declared it a pandemic. There was great concern, as there was still no knowledge about this new pathology and many countries did not have a strategic health plan. Therefore, this article aims to verify the efficacy of this drug in clinical studies. It is a systematic review, using the Preferred Reporting Items for Systematic Reviews and Meta-Analyses (PRISMA) protocol. The search strategy was designed using following controlled descriptors: Medical Subject Headings (MeSH) and Health Science Descriptors (DeCS) resources. The combinations of the descriptors resulted in the following terms: "Molnupiravir", "Covid-19" and "Sarscov-2". In addition, the Boolean operators "OR" and "AND" were applied. The databases used were Medline, Lilacs, both via the Virtual Health Library (VHL); PUBMED; Scientific Electronic Library Online (Scielo); Science Direct and Google Scholar. Molnupiravir can be used as a prophylactic measure, thus preventing hospitalization; in hospitalized patients with comorbidities, this drug has no clinical benefit. SInce the sample sizes in the studies were averagely low, there is a need for further studies to be carried out and replicated to verify if molnupiravir is effective against Covid-19.
\end{abstract}

Keywords: Covid-19; Molnupirair; SARS-CoV-2.

\title{
Resumen
}

Al inicio de 2020, el brote de (SARS-CoV-2), causante de la Covid-19, se diseminó rápidamente por el mundo, tanto que la Organización Mundial de la Salud (OMS) declaró estado de pandemia. Hube una gran preocupación, puesto que aún no se tenía conocimiento acerca de esa nueva patología y los países no tenían un plano de salud estratégico. Frente a esto, este artigo tiene el objetivo de verificar la eficacia de ese medicamento en estudios clínicos. Se trata de una revisión sistemática, se utilizó del protocolo Preferred Reporting Items for Systematic Reviwes and MetaAnalyses (PRISMA). La estrategia de busca fue construida a través de los descriptores controlados del Medical Subject Headings (MeSH) y de los recursos de los Descriptores en Ciencia de la Salud (DeCS). Las combinaciones de los descriptores han resultado en los siguientes términos: "Molnupiravir", "Covid-19" y "Sars-cov-2". Además, fueran aplicados los operadores booleanos "OR" y “AND”. Las bases de dados empleadas fueran Medline, Lilacs, ambas vía Biblioteca Virtual de la Salud (BVS); PUBMED; Scientific Eletronic Library Online (Scielo); Science Direct y Google Scholar. El molnupiravir puede ser empleado como medida profiláctica, por lo tanto, evitando la hospitalización, ya en pacientes internados y que poseían comorbilidades, el medicamento no tenía efecto clínico benéfico. Como las muestras en los estudios, en promedio, fueran bajas, es necesario que más estudios sigan siendo hechos y reproducidos para comprobar si el molnupiravir tiene eficacia en el combate al Covid-19.

Palabras clave: Covid-19; Molnupirair; SARS-CoV-2.

\section{Introdução}

No início de 2020, o surto de (SARS-CoV-2), causador da Covid-19, disseminou-se rapidamente pelo mundo, tanto que a Organização Mundial da Saúde (OMS) declarou estado de pandemia. Houve uma grande preocupação, pois ainda não se tinha conhecimento sobre essa nova patologia e os países não tinham um plano de saúde estratégico e, segundo a OMS, em março do mesmo ano, já havia ultrapassado 214 mil casos em todo o mundo (Freitas et al., 2020; Who., 2020).

A pandemia foi considerada uma ameaça à vida das pessoas, já que a Covid-19 apresenta uma taxa de letalidade mais alta do que a gripe A, uma vez que a responsável pela tal situação pandêmica possui uma taxa de incubação de 1 a 14 dias e é transmitida comunitariamente, por meio de gotículas contaminadas de secreções da orofaringe, de contato com superfícies e objetos contaminados, nos quais o vírus pode permanecer por até 3 dias e de livre circulação de pessoas assintomáticas. Apesar de a letalidade ser menor do que a de outros coronavírus, a doença pode promover a superlotação dos leitos de unidade de terapia intensiva, o que pode afetar os sistemas de saúde público e privado. Como a taxa de propagação ocorre em uma velocidade maior que a capacidade dos hospitais oferecem, provocaram-se crises sanitárias e econômicas nos países afetados (Nunes, 2020; Alquino et al., 2020; Estevão, 2020).

De acordo com a OMS, o padrão ouro de diagnóstico para a Covid-19, é o RT-PCR, que envolve técnicas de reação em cadeia da polimerase com transcrição reversa com amplificação em tempo real. A amostra pode ser obtida por secreção respiratória ou espirado nasofaríngeo (ANF) após o surgimento dos sintomas, entre o terceiro e o quinto dia (Nogueira \& Silva, 
2021). O exame de RT-PCR pode ser feito também de modo caseiro através de conjuntos diagnosticados (kits). Diversos países possuem protocolos de RT-PCR e, no Brasil, várias empresas registraram kits para o autodiagnóstico de SARS-CoV-2 por RTPCR na Agencia Nacional de Vigilância Sanitária (Anvisa) (Menezes, Lima \& Martinello, 2021).

O SARS-CoV-2 infecta o trato respiratório, havendo um possível estado crítico de vários pacientes. Sendo assim, o referido fato acontece porque o vírus infecta as células dos sistema imune, a saber: macrófagos, células dendríticas, linfócitos e monócitos, promovendo uma cascata inflamatória, pela liberação de citocinas inflamatórias, como IL2, IL7, IL10, GCSF, IP10MCP!, MIP1A e TNF-alfa, de forma exacerbada, o que causa a atração de leucócitos para o sítio inflamatório, lesionando os pneumócitos (CARVALHO et al., 2020). Ademais, modifica-se a estrutura do alvéolo, de tal forma que surgem dificuldades no processo de troca gasosa, resultando em hipoxemia nos pacientes e fibrose nos bronquíolos terminais e nas paredes alveolares (Souza Carvalho et al., 2021; Huang et al., 2020).

Em relação aos exames radiológicos, a radiografia e a tomografia computadorizada (TC) podem ser utilizados para o acompanhamento do paciente. Entretanto, a radiografia possui menor sensibilidade em relação à TC, cuja característica é um desempenho proveitoso, pelo fato de apresentar uma alta resolução e uma sensibilidade impecável. Os achados clínicos na TC costumam ser opacidade em vidro fosco e consolidações, principalmente em áreas periféricas, danificando as regiões posteriores do pulmão. Além disso, pode ser encontrado sinal do halo invertido e pavimentação em mosaico (Valentin et al., 2020; Lima, 2020).

Os principais sinais clínicos incluem: tosse, mialgia, leucopenia, linfopenia e febre. A maiorias das pessoas apresentam sintomas leves, não obstante, em indivíduos que apresentem comorbidades, o quadro clinico tende a se apresentar de forma mais grave (Naicker et al., 2020). Outros sintomas diferentes podem aparecer, como anosmia, conjuntivite, manifestações cutâneas alterações renais, dor abdominal e complicações na gravidez (Monte et al., 2020).

No público pediatra, há evidências e relatos sobre a COVID-19, mas ainda não há muitas informações concretas para serem discutidas. Apesar de a doença provocar poucos números de óbitos em comparação com os adultos, e de as crianças tenderem a desenvolver a forma, elas tornam-se reservatórios virais, mesmo sem apresentar sintomas, o que pode promover a disseminação do vírus para outras pessoas que fazem parte do grupo risco, por exemplo, idosos, pessoas com comorbidades, imunossuprimidos, pacientes oncológicos e cardiopatas (Monte et al.,2020).

A pandemia do novo coronavírus, portanto, tem provocado diversas mortes, sequelas em pacientes recuperados e, além disso, crises financeiras. $\mathrm{O}$ atual panorama exige que novos tratamentos sejam implementados para que esse cenário devastador cesse. Diante disso, é importante frisar que o molnupiravir é um medicamento antiviral e surgiu como um possível tratamento para pacientes infectados não-hospitalizados e hospitalizados. Por conseguinte, este artigo tem o objetivo de verificar a eficácia desse medicamento em estudos clínicos e, a partir disso, extrair as principais informações observadas nas pesquisas.

\section{Metodologia}

Este artigo trata-se de uma de revisão sistemática, que é uma modalidade de pesquisa que segue protocolos específicos, cujo objetivo é entender e seguir uma linha de raciocínio diante de um corpus documental, buscando, então, verificar o que funciona e o que não funciona em um determinado contexto. Sendo assim, possui como prioridade o foco na capacidade de reprodutibilidade por outros pesquisadores.

Esta pesquisa, portanto, apresenta, de forma transparente, as bases de dados, os critérios de inclusão e exclusão e o processo de análise de artigos (Galvão \& Ricarte, 2019). Utilizou-se do protocolo Preferred Reporting Items for Systematic Reviwes anda Meta-Analyses (PRISMA) (Page et al., 2021), o qual foi registrado no International Prospective Register of Systema Reviews (PROSPERO), sob o número CRD42022308474, seguindo as recomendações internacionais (Prospero, 2021).

Para auxiliar na elaboração da pergunta norteadora e na escolha dos critérios de elegibilidade, foi utilizado o acrômio PICO (Quadro 1). 
Quadro 1. PICO estratégia de investigação.

\begin{tabular}{|c|ll|}
\hline Acrônimo & \multicolumn{1}{|c|}{ Descrição } & \multicolumn{1}{c|}{ Definição } \\
\hline P & Pacientes & Adultos com COVID-19 \\
\cline { 1 - 1 } & Internação & Uso de molnupiravir \\
\hline Controle & Grupo placebo \\
\cline { 1 - 2 } & Resultado & Eficácia do molnupiravir - \\
& & diminuição da taxa de óbito \\
\hline
\end{tabular}

Fonte: Autores.

A partir disso, o estudo foi guiado pela seguinte questão norteadora: "O uso de molnupiravir é eficaz no tratamento contra COVID-19 em pacientes diagnosticados?" Seguindo tal perspectiva, a estratégia de busca foi construída através os descritores controlados do Medical Subject Headings (MeSH) e dos recursos dos Descritores em Ciência da Saúde (DeCS). As combinações dos descritrores resultaram nos seguintes termos: "Molnupiravir", "COVID-19" e "Sars-cov-2". Ademais, foram aplicados os operadores booleanos "OR" e "AND". As bases de dados utilizadas foram Medline, Lilacs, ambas via Biblioteca Virtual da Saúde (BVS); PUBMED; Scientific Eletronic Library Online (Scielo); Science Direct e Google Scholar.

Durante a revisão sistemática, foram considerados como critérios de inclusão: artigos publicados nos idiomas: português, inglês ou espanhol disponíveis na íntegra; ensaios clínicos randomizados; duplos-cegos; com limitação de ano, de 2020 a 2022, e estudos que tivessem como objetivo a eficácia do molnupiravir em pacientes com COVID-19. Os critérios de exclusão foram: artigos de revisão, textos incompletos, notícias, teses, monografias, intervenção em animais e estudos que tangenciaram o tema e temas não relacionados com o objetivo desta pesquisa.

A priori, houve a procura dos artigos durante o mês de fevereiro de 2022 e, logo após, foi realizada, por três revisores, a leitura dos resumos. Na referida etapa, excluíram-se os artigos incompletos ou que não se encaixaram no tema proposto. A partir de então, os artigos selecionados foram baixados para a leitura na íntegra com o intuito de obter as informações que alimentaram tabelas do programa Microsoft Excel. É importante destacar que o software Mendely foi imprescindível para a exclusão dos artigos duplicados. Ademais, dois revisores, de forma independente, avaliaram os artigos selecionados e obtiveram as informações necessárias para a constituição da tabela final. Destarte, os dados foram comparados e discutidos. A figura 1 mostra o fluxograma baseado no método PRISMA utilizado nesta pesquisa. 
Figura 1-Fluxograma baseado no método PRISMA.

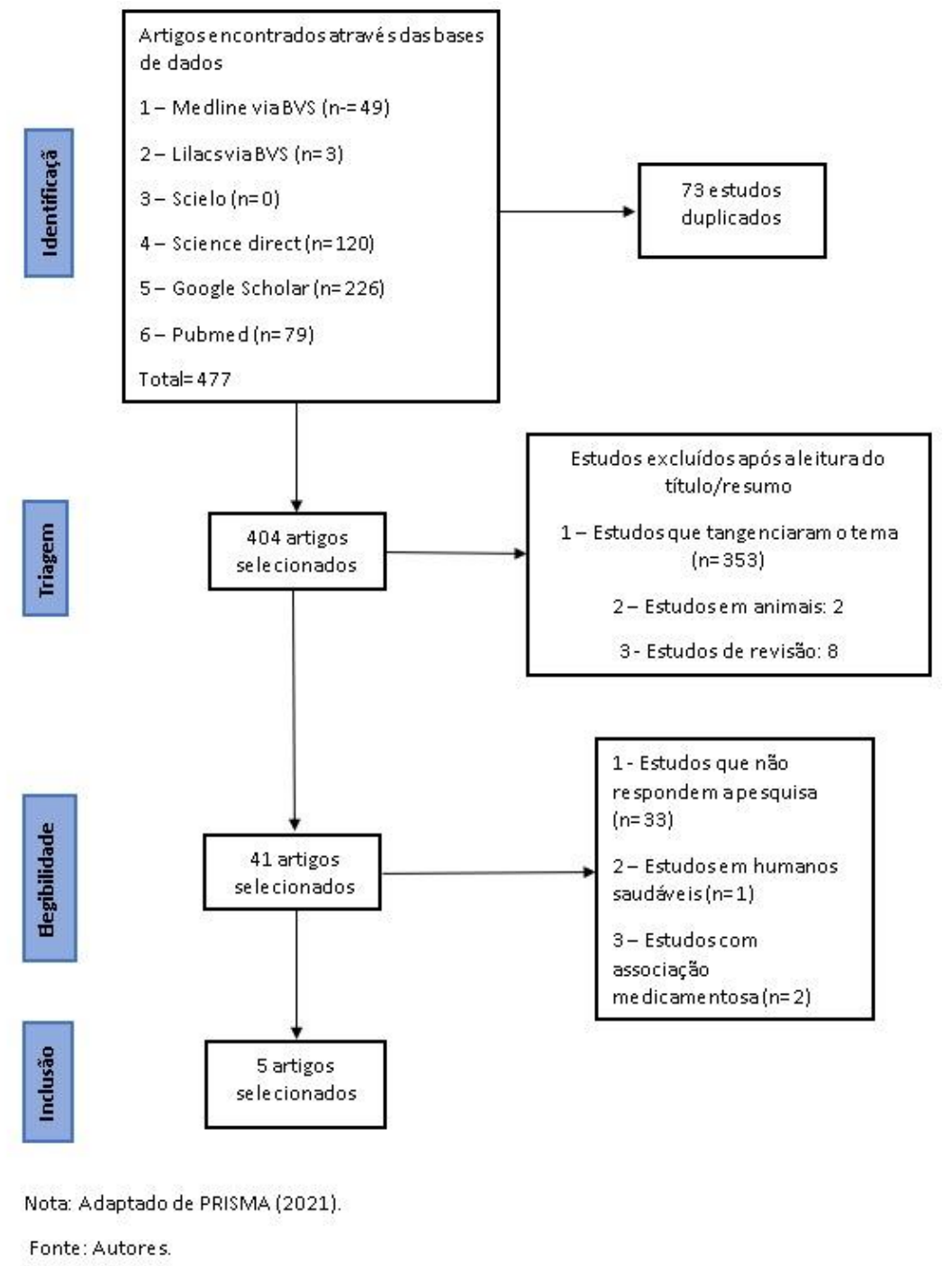

\section{Resultados e Discussão}

Após a aplicação dos descritores nas bases de dados e dos critérios de inclusão e exclusão, foi encontrado no total 477 artigos, sendo que 5 artigos foram selecionados (Quadro 2). Todos estão no idioma inglês. 
Quadro 2 - Dados gerais do Estudo.

\begin{tabular}{|c|c|c|c|c|}
\hline $\begin{array}{c}\text { AUTOR } \\
\text { PRINCIPAL E } \\
\text { ANO }\end{array}$ & TÍTULO & $\begin{array}{c}\text { AMOST } \\
\text { RA }\end{array}$ & $\begin{array}{c}\text { CONCEPÇÃO DO } \\
\text { ESTUDO }\end{array}$ & PRINCIPAIS RESULTADOS \\
\hline $\begin{array}{l}\text { A. Jayk Bernal } e t \\
\text { al., } 2021\end{array}$ & $\begin{array}{l}\text { Molnupiravir for Oral } \\
\text { Treatment of Covid-19 } \\
\text { in } \\
\text { Nonhospitalized Patients }\end{array}$ & 1411 & $\begin{array}{l}\text { É um estudo contínuo, } \\
\text { fase } 2 / 3 \text {, aleatório, } \\
\text { controlado por placebo, } \\
\text { duplo-cego. }\end{array}$ & $\begin{array}{l}\text { O tratamento precoce com molnupiravir } \\
\text { reduziu o risco de hospitalização ou } \\
\text { risco de morte adultos não vacinados } \\
\text { com COVID-19. }\end{array}$ \\
\hline
\end{tabular}

Saye H. Khoo et al.,2021

William A.

Fischer II et al. 2022

$\begin{array}{ll}\text { Yoseph Caraco et } & \text { Phase 2/3 Trial of } \\ \text { al., 2021 } & \text { Molnupiravir for Treatment } \\ & \text { of } \\ & \text { Covid-19 in } \\ & \text { Nonhospitalized Adults }\end{array}$

Jose R. Arribas et Randomized Trial of al., $2021 \quad$ Molnupiravir or Placebo in Patients Hospitalized with Covid-19 SARS-CoV-2: a Phase I, open-label, dose-escalating, randomized controlled study
Estudo de fase 1 de doseescalação, randomizado, controlado.

202

Estudo de fase 2, duplocego, controlado por placebo, randomizado.

308

Estudo de fase $2 / 3$, randomizado, controlado por placebo, duplo-cego.

304

Estudo de fase 2/3, duplocego, randomizado, controlado por placebo.
Molnupiravir foi considerado seguro e bem tolerado; foi recomendada uma dose de $800 \mathrm{mg}$ duas vezes por dia durante 5 dias para avaliação da fase 2 .

\begin{abstract}
Os resultados desse ensaio demonstram segurança, tolerabilidade e eficácia antiviral do molnupiravir para reduzir a replicação do SRA-CoV-2 e acelerar a eliminação do vírus infeccioso. Apoia os ensaios clínicos em curso do molnupiravir.
\end{abstract}

Resultados de estudos provisórios apoiam uma avaliação adicional do molnupiravir como tratamento potencial para reduzir hospitalizações e/ou morte em doentes não hospitalizados com COVID-19.

Não demonstrou benefício clínico.

Fonte: Autores.

Retomando o nosso assunto propulsor do presente estudo, é asseverado que pandemia do novo coronavírus foi declarada no início do ano de 2020 pela Organização Mundial de Saúde (OMS) e mobilizou instituições públicas e privadas em nível global para combatê-la (Freitas et al.,2020). O medicamento molnupiravir foi um dos recursos escolhidos, que ainda se encontra em estudos de ensaios clínicos, para validar sua eficácia no tratamento contra a Covid-19. O mesmo mostrou-se como um potencial recurso para pacientes diagnosticados com Covid-19, confirmados em laboratório, em pacientes não hospitalizados com sete dias (Caraco et al., 2021) e cinco dias (Bernal et al., 2021) do início dos sintomas, antes da intervenção.

Nos embasando no estudo de Caraco et al., foi possível depreender que os participantes, os quais também incluíam grupos de risco, foram aleatorizados por um sistema de tecnologia de resposta interativa para receber placebo $(\mathrm{n}=74), 200 \mathrm{mg}$ $(\mathrm{n}=75), 400 \mathrm{mg}(\mathrm{n}=77)$ ou $800 \mathrm{mg}(\mathrm{n}=76)$ do medicamento duas vezes ao dia durante um período de cinco dias e monitorados ao longo do tempo, durante vinte e nove dias. Dentre os pacientes que receberam a dose de $400 \mathrm{mg}$, um teve que ser hospitalizado e recebeu cinco doses ou menos; por outro lado, os que receberam a dose $800 \mathrm{mg}$, três foram hospitalizados e também receberam cinco doses ou menos. No entanto, os que receberam mais de cinco doses, um no grupo de $200 \mathrm{mg}$ e dois no de $400 \mathrm{mg}$, precisaram ser hospitalizados. No grupo controle, quatro precisaram de intervenções médicas. 
Nesse estudo, os autores relataram que não ocorreram anomalias significativas nos parâmetros hematológicos, hepáticos ou pancreáticos em decorrência das doses administradas e, além disso, não houve efeitos de toxicidade nem efeitos adversos na dose máxima ( $800 \mathrm{mg}$ ). Outrossim, no grupo placebo, ocorreu um óbito no total de 74 participantes e, no grupo de intervenção, houve um óbito no total de 228 participantes.

Ancorando-se na pesquisa realizada por Bernal et al., que consiste na fase 3 do estudo supracitado, houve, da mesma forma, a avaliação em pacientes não hospitalizados, envolvendo pessoas que não estavam vacinadas, 17,8\% da amostra. A dose utilizada foi a limite $(800 \mathrm{mg})$, durante vinte e nove dias, com uma amostra de $\mathrm{n}=1411$. Foram relatados efeitos adversos no grupo de intervenção e no grupo placebo, todavia, o monulpiravir mostrou benefícios com tratamento continuo em outras variantes do Sars-Cov-2, como delta e gama.

O fármaco também se mostrou ser eficaz como medida de profilaxia, o que reduziu o número de hospitalizações e óbitos. No grupo de pacientes que receberam as doses, o número de óbitos, $n=2$, foi baixo quando comparado com o grupo placebo, $n=12$. Em suma, quando iniciado cinco dias após o início dos sinais e/ou sintomas, demonstrou segurança com a dose limite e eficácia em pacientes que pertenciam ao grupo de risco.

Na pesquisa realizada por Khool et al., o tamanho da amostra não permitiu realizar estatisticamente a eficácia do medicamento, apenas demonstrou que a dose de $800 \mathrm{mg}$ foi bem tolerada e houve alguns efeitos adversos leves, como dor abdominal e diarreia. Sendo assim, o estudo prosseguiu para a segunda fase.

No estudo na fase 2 de Fischer et al., apresenta-se uma amostra de 202 pacientes não vacinados. Sendo assim, foram utilizadas doses de $200 \mathrm{mg}, 400 \mathrm{mg}$ e $800 \mathrm{mg}$, durante sete dias após o surgimento dos primeiros sintomas e acompanhamento durante vinte e oito dias. Por conseguinte, em todos os grupos que receberam as doses, não foram registrados mortes causadas pela Covid-19 ou outro efeito adverso, ao contrário do grupo placebo, que registrou uma morte. A análise estatística evidenciou que o molnupiravir foi bem tolerado e considerado eficaz para redução da carga viral, corroborando com os achados de (Caraco et al., 2021; Bernal et al., 2021).

Todavia, os achados de Arribas et al. são discrepantes em relação aos resultados dos estudos que mostraram benefícios com o uso do molnupiravir. Os participantes eram pacientes adultos hospitalizados e receberam doses de $200 \mathrm{mg}, 400 \mathrm{mg}$ e 800 mg, no entanto, não mostraram benefícios clínicos. Os participantes não estavam vacinados, visto que a vacinação era um critério de exclusão do estudo, e apresentavam fatores de risco para o desenvolvimento da forma grave da doença, como diabetes e a idade superior a sessenta anos. No total, ocorreram vinte e oito óbitos no grupo de intervenção e duas mortes no grupo controle.

Em comparação com um ensaio-clínico realizado em hamsters fêmeas, por Abdelnabi et al., o monulpiravir mostrou ser eficiente na redução da carga viral, estatisticamente, em cada variante e que pode ser utilizado em futuras mutações que tendem a acontecer. Em um outro estudo realizado pelos mesmos autores Abdelnabi et al., também em hamsters, fez-se uma associação do monulpiravir com o favipiravir, o que resultou na produção de um antiviral com potencial para inibir a replicação do vírus. Logo, com base nisso, essa combinação poder ser usada em humanos para combater a pandemia do novo coronavírus.

\section{Conclusão}

A pandemia do novo coronavírus promoveu diversas mortes e crises econômicas ao redor do mundo. Para combater esse cenário de caos, vários medicamentos foram selecionados como possíveis soluções para o tratamento em humanos infectados. Dentre esses medicamentos, está o molnupiravir, um antiviral possivelmente capaz de reduzir a carga viral dessa patologia. Nos estudos analisados, ele apresentou mais eficácia em pacientes não hospitalizados que possuíam fatores de risco ou não para desenvolver a forma grave, com início dos sintomas dentro de cinco a sete dias. Dessa forma, o molnupiravir pode ser usado como medida de profilaxia, sendo assim, evitando a hospitalização.

Ademais, já em pacientes hospitalizados e que possuíam comorbidades, o medicamento não possui efeito benéfico clínico. Como as amostras nos estudos, em média, foram baixas, é necessário que mais estudos continuem sendo feitos e 
reproduzidos para comprovar se o molnupiravir possui eficácia no combate à pandemia em pacientes vacinados e não vacinados, com comorbidades e também no público pediátrico, uma vez que este não foi incluso nos estudos dos artigos selecionados.

Em suma, os futuros trabalhos a serem realizados devem incluir o público pediátrico para que se possa saber se o medicamento possui algum efeito sobre as crianças. Além disso, deverão envolver uma amostra com a maior quantidade possível de pacientes, diagnósticos com Covid-19, para que se possa realizar uma análise estatística para obter dados quantitativos fidedignos com a realidade. Outrossim, determinar a dose, com desenhos estudos adequados, para que se possa oferecer uma melhor qualidade de tratamento ao paciente.

\section{Referências}

Abdelnabi, R., Foo, C. S., De Jonghe, S., Maes, P., Weynand, B., \& Neyts, J. (2021). Molnupiravir Inhibits Replication of the Emerging SARS-CoV-2 Variants of Concern in a Hamster Infection Model. The Journal of infectious diseases, 224(5), 749-753. https://doi.org/10.1093/infdis/jiab361

Abdelnabi, R., Foo, C. S., Kaptein, S., Zhang, X., Do, T., Langendries, L., Vangeel, L., Breuer, J., Pang, J., Williams, R., Vergote, V., Heylen, E., Leyssen, P., Dallmeier, K., Coelmont, L., Chatterjee, A. K., Mols, R., Augustijns, P., De Jonghe, S., Jochmans, D., ... Neyts, J. (2021). The combined treatment of Molnupiravir and Favipiravir results in a potentiation of antiviral efficacy in a SARS-CoV-2 hamster infection model. EBioMedicine, 72, 103595. https://doi.org/10.1016/j.ebiom.2021.103595

Aquino, E. M., Silveira, I. H., Pescarini, J. M., Aquino, R., Souza-Filho, J. A. D., Rocha, A. D. S., ... \& Lima, R. T. D. R. S. (2020). Medidas de distanciamento social no controle da pandemia de COVID-19: potenciais impactos e desafios no Brasil. Ciência \& Saúde Coletiva, 25(suppl 1), $2423-2446$.

Arribas, J. R., Bhagani, S., Lobo, S. M., Khaertynova, I., Mateu, L., Fishchuk, R., ... \& De Anda, C. (2022). Randomized trial of molnupiravir or placebo in $\begin{array}{llll}\text { patients } & \text { with } & \text { Covid-19. NEJM } & \text { Evidence, 1(2), }\end{array}$ Caraco, Y., Crofoot, G. E., Moncada, P. A., Galustyan, A. N., Musungaie, D. B., Payne, B., ... \& De Anda, C. (2022). Phase 2/3 trial of molnupiravir for treatment of Covid-19 in nonhospitalized Evidence, 1(2), adults. NEJM

Souza Carvalho, F. R., Gobbi, L. C., Carrijo-Carvalho, L. C., Caetano, A. J. F., Casotti, G. C., Tiussi, L. M., ... \& Cavalari, A. L. C. (2020). Fisiopatologia da COVID-19: repercussões sistêmicas. Unesc em Revista, 4(2), 170-184.

Galvão, M. C. B., \& Ricarte, I. L. M. (2019). REVISÃO SISTEMÁTICA DA LITERATURA: CONCEITUAÇÃO, PRODUÇÃO E PUBLICAÇÃO. Logeion:

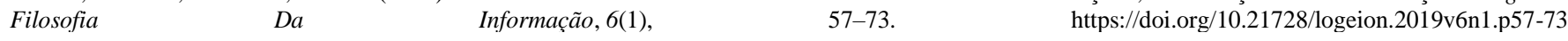

Huang, R., Xia, J., Chen, Y., Shan, C., \& Wu, C. (2020). A family cluster of SARS-CoV-2 infection involving 11 patients in Nanjing, China. The Lancet.

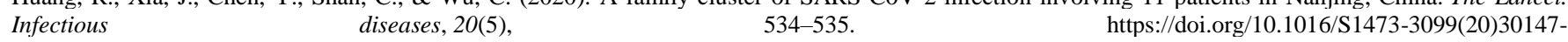

Jayk Bernal, A., Gomes da Silva, M. M., Musungaie, D. B., Kovalchuk, E., Gonzalez, A., Delos Reyes, V., Martín-Quirós, A., Caraco, Y., Williams-Diaz, A., Brown, M. L., Du, J., Pedley, A., Assaid, C., Strizki, J., Grobler, J. A., Shamsuddin, H. H., Tipping, R., Wan, H., Paschke, A., Butterton, J. R., ... MOVe-OUT Study Group (2022). Molnupiravir for Oral Treatment of Covid-19 in Nonhospitalized Patients. The New England journal of medicine, 386(6), 509-520. https://doi.org/10.1056/NEJMoa2116044.

Khoo, S. H., Fitzgerald, R., Fletcher, T., Ewings, S., Jaki, T., Lyon, R., Downs, N., Walker, L., Tansley-Hancock, O., Greenhalf, W., Woods, C., Reynolds, H., Marwood, E., Mozgunov, P., Adams, E., Bullock, K., Holman, W., Bula, M. D., Gibney, J. L., Saunders, G., ... Griffiths, G. (2021). Optimal dose and safety of molnupiravir in patients with early SARS-CoV-2: a Phase I, open-label, dose-escalating, randomized controlled study. The Journal of antimicrobial chemotherapy, 76(12), 3286-3295. https://doi.org/10.1093/jac/dkab318

Lima, F. L. O., Gomes, L. N. L., Santos, C. S. C. d., \& Oliveira, G. A. L. d. (2020). Diagnóstico da COVID-19: Importância dos testes laboratoriais e dos exames de imagem. Research, Society and Development,

Menezes, M. E., Lima, L., \& Martinello, F. (2020). Diagnóstico laboratorial do SARS-CoV-2 por transcrição reversa seguida de reação em cadeia da polimerase em tempo real (RT-PCR). A Rempestade do Rev Coronavirus.

MonteL. M. do, MendesL. A., CamargoR. L., GomesR. S. de S., da SilveiraP. H. A., SeyfarthM. S. C., CunhaD. M., OliveiraL. de P. R., da SilveiraR., \& da SilveiraG. R. R. A. (2020). Complicações atípicas e características clínico-epidemiológicas do COVID-19: uma revisão integrativa. Revista Eletrônica Acervo Saúde,

$$
\text { (46), e3699. }
$$
https://doi.org/10.25248/reas.e3699.2020

PROSPERO,(2021). Prospero National Institute for Health Research.https://www.crd.york.ac.uk/prospero/

Rocha Nogueira, J. M. (2020). Diagnóstico laboratorial da Covid-19 no Brasil. A Tempestade do Coronavírus, 52(2), 117-21. Nunes, J. (2020). A pandemia de COVID-19: securitização, crise neoliberal e a vulnerabilização global. Cadernos de Saúde Pública, 36.

Page, M. J., McKenzie, J. E., Bossuyt, P. M., Boutron, I., Hoffmann, T. C., Mulrow, C. D., Shamseer, L., Tetzlaff, J. M., Akl, E. A., Brennan, S. E., Chou, R., Glanville, J., Grimshaw, J. M., Hróbjartsson, A., Lalu, M. M., Li, T., Loder, E. W., Mayo-Wilson, E., McDonald, S., McGuinness, L. A., ... Moher, D. (2021). The PRISMA 2020 statement: an updated guideline for reporting systematic reviews. BMJ (Clinical research ed.), 372, n71. https://doi.org/10.1136/bmj.n71

Naicker, S., Yang, C. W., Hwang, S. J., Liu, B. C., Chen, J. H., \& Jha, V. (2020). The Novel Coronavirus 2019 epidemic and kidneys. Kidney international, 97(5), 824-828. https://doi.org/10.1016/j.kint.2020.03.001 
Research, Society and Development, v. 11, n. 3, e44211326902, 2022

(CC BY 4.0) | ISSN 2525-3409 | DOI: http://dx.doi.org/10.33448/rsd-v11i3.26902

WHO (WORLD HEALTH $\quad$ ORGANIZATION).2020a.Coronavirus $\quad$ disease $2019 \quad$ (COVID-19) reports.https://www.who.int/emergencies/diseases/novel

ValentinM. V., DiasC. C., BarbosaH. L. M., \& BragaD. O. (2020). Aspectos radiológicos em paciente com covid-19: um relato de caso. Revista Interdisciplinar De Saúde E Educação, 1(2), 249-259. Recuperado de https://periodicos.baraodemaua.br/index.php/cse/article/view/103

Estevão,A.(2020).

COVID-19. Acta

Radiológica

Portuguesa, 32(1),

$5-6$

Fischer, W. A., 2nd, Eron, J. J., Jr, Holman, W., Cohen, M. S., Fang, L., Szewczyk, L. J., Sheahan, T. P., Baric, R., Mollan, K. R., Wolfe, C. R., Duke, E. R., Azizad, M. M., Borroto-Esoda, K., Wohl, D. A., Coombs, R. W., James Loftis, A., Alabanza, P., Lipansky, F., \& Painter, W. P. (2022). A phase 2a clinical trial of molnupiravir in patients with COVID-19 shows accelerated SARS-CoV-2 RNA clearance and elimination of infectious virus. Science translational medicine, 14(628), eabl7430. https://doi.org/10.1126/scitranslmed.abl7430

Freitas, A. R. R., Napimoga, M., \& Donalisio, M. R. (2020). Análise da gravidade da pandemia de Covid-19. Epidemiologia e serviços de saúde, 29 , e2020119. 